\title{
Smart Wells Optimization in Oil Reservoirs Using Adjoint Gradient Model
}

\author{
Xian Shan \\ College of Science \\ China University of Petroleum (East China) \\ Qingdao, China \\ e-mail: shanxianlw@163.com
}

\author{
Tao Zhang \\ School of Economics and Management \\ China University of Petroleum (East China) \\ Qingdao, China \\ e-mail: zhangtao@upc.edu.cn
}

\begin{abstract}
Smart wells can effectivelyimprove the reservoir developing by selectively controlling flow production of the valves. In this work, we proposed an adjoint-based gradient method for the smart well control optimization within the search for optimum smart wells inflow control valves configuration. Numerical study showed that the NPV value can be increased obviously by the optimization. The displacement efficiency can also be improved.
\end{abstract}

Keywords- smart wells; adjoint-based gradient; NPV value;optimization

\section{INTRODUCTION}

Smart wells are wells equipped with intelligent completion, which has packers or sealing elements allowing partitioning of the wellbore. They have enormous advantage in the reservoir developing. By selectively controlling production from flow of the valves, smart wells can delay the water breakthrough which is along the high-permeability channels, and at the same time improve the reservoir production. In the successful application of smart well,one of the key issues technology is the valve flow optimized decision.

There are two principal methods to solvethe optimal control of smart wells' valve flow in the mathematical model:

(1) Stochastic algorithms, such as geneticalgorithm (Alghareeb et al.[1]), Ensemble Kalman filter methods (Lorentzen etal.[2]), and derivative-free algorithm (Zhao et al.[3]). These methods are simple with highaccuracy, but the computational cost increases rapidly with the increase of variables, which limits their practicalapplication.

(2) Gradient-based algorithms.In the optimal model, calculation of the gradient is based on the maximum principle (Brouwer[4], Jansen et al.[5] and Zhang et al.[6]). The algorithms make higher efficiency. However, they are more complex than other methods due to the complexity of the adjoint equation.

This paper describes the smart well production optimization model as an augmented Lagrangian problem, and puts forward an improved method, combined with the forward reservoir simulation model and the backward adjoint gradient calculation, to solve the problem. Numerical results demonstrating the capabilities of our optimization procedures are provided in the last section.

\section{PROBLEM DESCRIPTION AND APPROACHES}

This study focuses on finding the optimum inflow control values configuration to maximize the economic revenue of production. Firstly, the economic revenue objective functionis detailed, which is depended on the injection and production rates. Then the governing equations that describe the reservoir flow process are presented, which allow the evaluation of the objective function.

\section{A. Economic model}

In general, the economic revenue objective function is defined as a function of the injection rates, production rates and the production time. It is often associated with oil prices, the costs of injection water and the costs of disposing produced water. Summing up the revenues over the time horizon $[0, T]$, the net-present-value (NPV) can be acquired as follows:

$$
\begin{aligned}
& N P V= \\
& \sum_{n=1}^{N_{f}}\left[\frac{\sum_{j=1}^{N_{p r d o}}\left(r_{o} q_{o, j}^{n}-r_{w} q_{w, j}^{n}\right)-\sum_{j=1}^{N_{i n j}}\left(r_{w, i n j} q_{i n j, i}^{n}\right)}{(1+b)^{t^{n}}}\right] \Delta t^{n}
\end{aligned}
$$

Where,

$N_{t}$ is the time steps of reservoir simulation,

$N_{i n j}$ is the number of the injection wells,

$N_{\text {pro }}$ is the number of the production wells,

$\Delta t^{n}$ is the size of the $n^{t h}$ time step in days,

$t^{n}$ is the total simulation time in days at the end of the $n^{\text {th }}$ time step,

$q_{o, j}^{n}$ is the oil production rates of the $j^{t h}$ producer over the $n^{\text {th }}$ simulation time step,

$q_{w, j}^{n}$ is the water production rates of the $j^{\text {th }}$ producer over the $n^{\text {th }}$ simulation time step,

$q_{i n j, i}^{n}$ is the injection rate of the $i^{t h}$ injection well over the $n^{\text {th }}$ simulation time step, 
$r_{o}$ in $\$ / b b l$ is the price of oil per unit volume,

$r_{w}$ in $\$ / \mathrm{bbl}$ is the cost of disposing water per unit volume,

$r_{w, i n j}$ in $\$ / b b l$ is the cost of injecting water per unit volume,

$\mathrm{b}$ is the annual discount rate.

\section{B. Oil reservoir model}

In this work, we assume the reservoir pressures are always above the bubble point pressure of the oil phase. With a two-phase, immiscible oil-water model, the reservoirs are formulated as a set of partial differential equations for the conservation of mass of oil and water phase. Combined with oil and water equations, the pressure equation is given as

$$
\begin{gathered}
\mathbf{v}=-\mathbf{K} \lambda_{t}(s) \nabla p \text {,in } \Omega \\
\nabla \cdot \mathbf{v}=q \text {,in } \Omega \\
\mathbf{v} \cdot \mathbf{n}=0, \text { on } \partial \Omega
\end{gathered}
$$

Where,

$\Omega$ is the oil reservoir region,

$\partial \Omega$ is the oil reservoir boundary,

$\mathbf{V}$ is the total velocity of oil and water,

$\mathbf{K}$ is the permeability tensor,

$p$ is the pressure of oil phase,

$q$ is the volumetric well rate,

$\lambda_{t}$ is the total mobility of the water and oil phase,

The saturation equation is described as

$$
\phi \frac{\partial s}{\partial t}+\nabla \cdot f_{w}(s) \mathbf{v}=q_{w}
$$

Where

$\phi$ is the porosity of the porous medium,

$S$ is the water saturation,

$f_{w}(s)$ is the water fractional flow,

$q_{w}$ is the volumetric water rate at the well.

The pressure equation is discretized by cell-centered finite difference approach in space, which is given by

$$
\mathbf{A}\left(s^{n-1}\right) \mathbf{p}^{n}=\mathbf{B} \mathbf{u}^{n}
$$

Where

$n$ isthe discretized simulation time step,

$A\left(s^{n-1}\right)$ is the two-point flux transmissibility over the $n^{\text {th }}$ simulation time step, which depends on the water saturation $s^{n-1}$ over the $(n-1)^{t h}$ simulation timestep,

$\mathbf{p}^{n}$ is the vector of grid block pressures over the $n^{\text {th }}$ simulation time step,

$$
\mathbf{u}^{n}=\left[q_{i n j, 1}^{n}, q_{i n j, 2}^{n}, \ldots, q_{i n j, N_{i n j}}^{n}, q_{p r o, 1}^{n}, q_{p r o, 2}^{n} \ldots q_{i r o 1, N_{p r o}}^{n}\right]^{T}
$$

is the control input as contained well rates over the $n^{\text {th }}$ simulation timestep. $q_{i n j, i}^{n}$ is the injection rate at each injector $i\left(i=1,2, \ldots N_{i n j}\right)$ over the $n^{\text {th }}$ simulation timestep, and $q_{p r o, j}^{n}$ is the total liquid production rate at each producer $j\left(j=1,2, \ldots N_{\text {pro }}\right)$ over the $n^{\text {th }}$ simulation timestep.

$\mathbf{B}$ is the arrangement matrix for the control input over the $n^{\text {th }}$ simulation timestep.

The saturation equation is discretized by an implicit finite volume scheme

$$
\mathbf{s}^{n}=\mathbf{s}^{n-1}+\Delta t^{n} \mathbf{D}_{P V}^{-1}\left(R\left(\mathbf{v}^{n}\right) f_{w}\left(\mathbf{S}^{n}\right)+\mathbf{q}\left(\mathbf{v}^{n}\right)\right)
$$

Where

$\Delta t^{n}$ is the time step,

$D_{p v}$ is the diagonal matrix containing the grid block pore volume,

$\mathbf{R}\left(\mathbf{v}^{n}\right)$ is the sparse flux matrix based on an upstream weighted discretization scheme,

$\mathbf{q}\left(\mathbf{v}^{n}\right)$ is the vector of positive sources (in this setting, water injection rates).

In this work, the pressure and saturation equationsare solved sequentially. The discrete state equations can be rewritten in an implicit form $g^{n}\left(\mathbf{x}^{n}, \mathbf{x}^{n-1}, \mathbf{u}^{n}\right)=0$ as

$$
\begin{aligned}
\mathbf{x}^{n} & =\left(\mathbf{p}^{n}, \mathbf{s}^{n}\right) \\
\mathbf{u}^{n} & =\left[q_{i n j, 1}^{n}, q_{i n j, 2}^{n}, \ldots, q_{i n j, N_{i n j}}^{n}, q_{p r o, 1}^{n}, q_{p r o, 2}^{n} \ldots q_{i r o 1, N_{p r o}}^{n}\right]^{T}
\end{aligned}
$$

An initial condition on the saturations, i.e., $\mathbf{s}^{0}$, is also needed. The state vectors and control input vectors are stacked for all time instances from $n=1, \ldots N$. Both producer wells and injector wells are covered by this formulation.

\section{The optimization problem}

The optimum inflow control values configuration problem is defined as a problem to maximize the revenue objective function $J(\mathbf{x}, \mathbf{u})$, which is defined by the net-present-value function.

$$
\begin{aligned}
J(\mathbf{x}, \mathbf{u}) & =N P V \\
& =\sum_{n=1}^{N}\left[\sum_{j=1}^{N_{\text {prdo }}}\left(r_{o} q_{o, j}^{n}-r_{w} q_{w, j}^{n}\right)-\sum_{j=1}^{N_{i n j}}\left(r_{\text {winj }} q_{i n j, i}^{n}\right)\right] \Delta t^{n} \\
& =\sum_{n=1}^{N} f^{n}\left(\mathbf{x}^{n}, \mathbf{u}^{n}\right) \Delta t^{n}
\end{aligned}
$$




$$
\mathbf{u}^{n}=\left[q_{i n j, 1}^{n}, q_{i n j, 2}^{n}, \ldots, q_{i n j, N_{i j j}}^{n}, q_{p r o, 1}^{n}, q_{p r o, 2}^{n} \ldots q_{i r o 1, N_{p r o}}^{n}\right]^{T}
$$

The constraints of the optimal control problem are pressure equations,saturation equations, andinitial condition, which can be concluded in governing equations.

We can define the optimal control problem as following

$$
\begin{gathered}
\min J(\mathbf{x}, \mathbf{u}) \\
\text { subject to } g^{n}\left(\mathbf{x}^{n}, \mathbf{x}^{n-1}, \mathbf{u}^{n}\right)=0 \\
\mathbf{x}_{0}=\mathbf{x}\left(t_{0}\right)
\end{gathered}
$$

\section{Optimization method}

To solve the optimal control problem, the maximum principleis implemented to maximize the NPV function $J(\mathbf{x}, \mathbf{u})$, which is subjected to the bound constraints on the optimization variables.

The augmented objective function $L$ can be constructed by "adjoining" the governing equations to the objective function $J(\mathbf{x}, \mathbf{u})$,

$$
\begin{aligned}
L & =\sum_{n=1}^{N}\left(\Delta t^{n} f^{n}\left(\mathbf{x}^{n}, \mathbf{u}^{n}\right)+\lambda^{n T} \mathbf{g}^{n}\left(x^{n}, x^{n-1}, u^{n}\right)\right) \\
& +\lambda^{0 T}\left(x_{0}-x^{0}\right)
\end{aligned}
$$

where

$\lambda^{n T}$ are the Lagrange multipliers.

The two objective functions $L$ and $J(\mathbf{x}, \mathbf{u})$ have the same extreme value. The extreme of $L$ is achieved when the first variation of $L$ is zero $(\delta L=0)$.

Taking the variation of $L$, and grouping terms multiplied by the same variation ( $\delta \mathbf{x}^{n}, \delta \mathbf{x}^{N}, \delta \mathbf{u}^{n}$ ), $\delta L$ can be written as:

$$
\begin{aligned}
& \delta L=\left(\Delta t^{N} \frac{\partial f^{N}}{\partial \mathbf{x}^{N}}+\lambda^{N T} \frac{\partial \mathbf{g}^{N}}{\partial \mathbf{x}^{N}}\right) \delta \mathbf{x}^{N}+ \\
& +\sum_{n=1}^{N-1}\left(\Delta t^{n} \frac{\partial f^{n}}{\partial \mathbf{x}^{n}}+\lambda^{(n+1) T} \frac{\partial \mathbf{g}^{n+1}}{\partial \mathbf{x}^{n}}+\lambda^{n T} \frac{\partial \mathbf{g}^{n}}{\partial \mathbf{x}^{n}}\right) \delta \mathbf{x}^{n} \\
& +\sum_{n=1}^{N}\left(\Delta t^{n} \frac{\partial f^{n}}{\partial \mathbf{u}^{n}}+\lambda^{n T} \frac{\partial \mathbf{g}^{n}}{\partial \mathbf{u}^{n}}\right) \delta \mathbf{u}^{n} \\
& +\left(x_{0}-x^{0}\right) \delta \lambda^{0 T}+\sum_{n=1}^{N} \mathbf{g}^{n T} \delta \lambda^{n T}
\end{aligned}
$$

To satisfy $\delta L=0$, the gradient of the objective function is needed with respect to the state variables $\delta L / \delta \mathbf{x}^{n}=0$ (for $n=1,2, \ldots N$ ) and the control variables $\delta L / \delta \mathbf{u}^{n}=0$.
In order to achieve $\delta L / \delta \mathbf{x}^{n}=0$ and $\delta L / \delta \mathbf{x}^{N}=0 \quad$ (for $n=1,2, \ldots N$ ), the Lagrange multipliers are the solutions of the following adjoint equations

$$
\begin{aligned}
& \Delta t^{n} \frac{\partial f^{n}}{\partial \mathbf{x}^{n}}+\lambda^{(n+1) T} \frac{\partial \mathbf{g}^{n+1}}{\partial \mathbf{x}^{n}}+\lambda^{n T} \frac{\partial \mathbf{g}^{n}}{\partial \mathbf{x}^{n}}=\mathbf{0}^{T} \\
& \Delta t^{N} \frac{\partial f^{N}}{\partial \mathbf{x}^{N}}+\lambda^{N T} \frac{\partial \mathbf{g}^{N}}{\partial \mathbf{x}^{N}}=\mathbf{0}^{T}
\end{aligned}
$$

With the Lagrange multipliers that satisfy the above linear equations, the variation $\delta L$ becomes

$$
\delta L=\sum_{n=1}^{N}\left(\Delta t^{n} \frac{\partial f^{n}}{\partial \mathbf{u}^{n}}+\lambda^{n T} \frac{\partial \mathbf{g}^{n}}{\partial \mathbf{u}^{n}}\right) \delta \mathbf{u}^{n}
$$

The gradient of the augmented objective function $L$, with respect to the controls variables is

$$
\delta L / \delta \mathbf{u}^{n}=\Delta t^{n} \frac{\partial f^{n}}{\partial \mathbf{u}^{n}}+\lambda^{n T} \frac{\partial \mathbf{g}^{n}}{\partial \mathbf{u}^{n}}, n=1,2, \ldots N
$$

By driving $\delta L / \delta \mathbf{u}^{n}=0$, the extreme of $L$ is achieved.

\section{E. Optimization algorithm}

Letting the subscript $\mathrm{k}$ be the outer iteration counter, the following algorithmis considered to solve this optimization problem.

Algorithm 1. The adjoint algorithm

1. Choose $\mathbf{u}^{0}=\left\{\mathbf{u}_{0}^{n}\right\}_{n=1}^{N}, \mathbf{x}_{0}^{0}$. For $k=1,2, \ldots$ do:

2. For $n=1,2, \ldots N$, find $\mathbf{x}_{k}^{n}$ such that $g^{n}\left(\mathbf{x}_{k}^{n}, \mathbf{x}_{k}^{n-1}, \mathbf{u}_{k-1}^{n}\right)=0$

3. Find $\lambda_{k}^{N}$ such that

$\Delta t^{N} \frac{\partial f^{N}}{\partial \mathbf{x}^{N}}+\lambda^{N T} \frac{\partial \mathbf{g}^{N}}{\partial \mathbf{x}^{N}}=\mathbf{0}^{T}$

4. Find $\lambda_{k}^{n} n=N-1, N-2, \ldots 1$ such that

$$
\Delta t^{n} \frac{\partial f^{n}}{\partial \mathbf{x}^{n}}+\lambda^{(n+1) T} \frac{\partial \mathbf{g}^{n+1}}{\partial \mathbf{x}^{n}}+\lambda^{n T} \frac{\partial \mathbf{g}^{n}}{\partial \mathbf{x}^{n}}=\mathbf{0}^{T}
$$

5. Find the gradient of $L$ as

$$
\delta L / \delta \mathbf{u}^{n}=\Delta t^{n} \frac{\partial f^{n}}{\partial \mathbf{u}^{n}}+\lambda^{n T} \frac{\partial \mathbf{g}^{n}}{\partial \mathbf{u}^{n}}
$$

Use this gradient in the LBFGS algorithm to find $\mathbf{u}_{k}=\left\{\mathbf{u}_{k}^{n}\right\}_{n=1}^{N}$. 


\section{CASE STUDY}

A rectangular, heterogeneous reservoir with no-flow boundaries is shown in Fig. 1. The model consists of $15 \times 15 \times 1$ grid blocks with an injector along the left hand side and a producer along the right hand side. Each well has 15 controllable segments, which can be controlled individually. The fluid system is in incompressible oil-water two phases. The relative permeability of each phase is defined by the Corey model. The absolute permeability field of the reservoir is illustrated in Fig. 1. There are two high permeability zones distribute along the vertical direction of the wells throughout the reservoir.

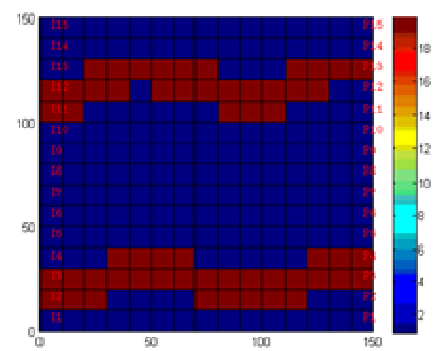

Figure 1. Permeability Field $(\mu \mathrm{m} 2)$

The total injection well rate is set to $200 \mathrm{~m}^{3} / \mathrm{d}$. The water injection and production of this reservoir do not change with time. The injection-production rates keepbalance. In the optimization problem, the control parameters are the well rates of injector segments and producer segments. The oil development period is 450 days. We update the production strategy every 45 days. Hence, in total, we have $(15+15) \times 10$ well rate control variables.

Initially, the total well rate is distributed in the 15 segments, according to the product of the absolute permeability and the net thickness in the segment grid. We run the simulation with the initial production strategy. The optimized production strategy is shown in Fig.2. It can be seen that the injection rates of the segments near the upper high permeability zoneare increased gradually from the first control step, which can delay the water fingering. The production rates of the segments near the lower high permeability zoneare set to a higher value, which can improve the displacement efficiency.

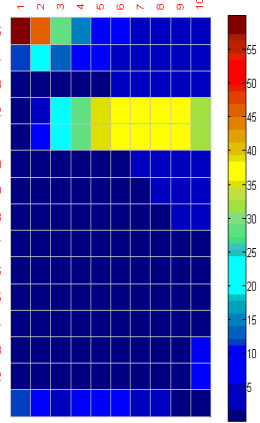

injectors

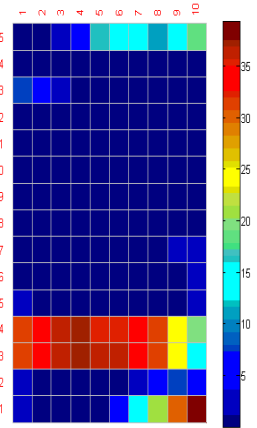

procucers
Figure 2. Water rate distribution in different control steps

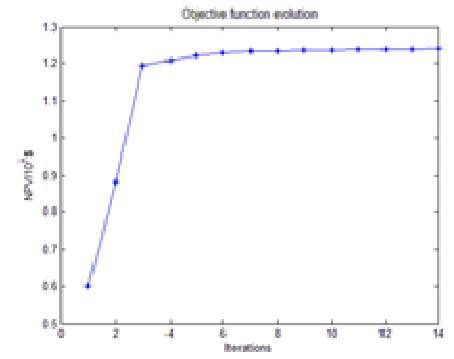

Figure 3. NPV in the optimization process

Figure 3shows that,the first iteration gets the largest growth of NPV. After 16 iterations, there is an increase of NPV from $0.59 \times 107 \$$ to $1.23 \times 107 \$$.

After 450 days production, water saturation fieldswith and without optimization are shown in Fig.4. We can see that, with the real-time control optimization, most of the reservoir oil can be displaced by water, and the sweep efficiency isimproved significantly.

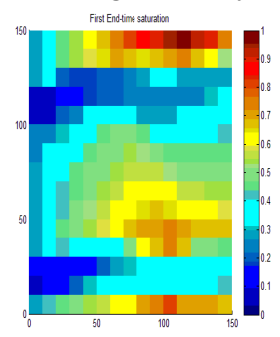

Before optimization

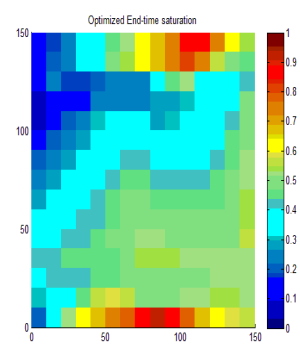

After optimization
Figure 4. Oil saturation distribution

\section{CONCLUSIONS}

(1) Combined with the fully implicit reservoir model as well as theaugmented Lagrangian function,asmart well control optimization model is established.

(2) The control optimization model isoptimized by the adjoint gradients method, whichcan calculate the gradient efficiently through the solution ofadjoint equation. The adjoint gradient algorithm can also be effectively used to constrain the NPV maximum problem, the filed total production maximum problem and other parameters optimum problems.

(3) In real oil reservoirs, with more complex objective functions and non-linear constrains, there are a number of decision variables in the field optimum problem. A generalized reservoir development optimization problem is still full of challenge. An idea for future direction is incorporating different techniques to handle non-linear systems.

\section{REFERENCES}

[1] Alghareeb Z. M., HORNE R. N. and YUEN B. B. Proactive optimization of oil recovery in multilateral wells using real time production data[C].SPE Annual Technical Conference and Exhibition, 124999-MS. New Orleans, Louisiana, USA, 2009.

[2]LORENTZEN R. J., BERG A. M. and NAEVDAL G. A new approach for dynamic optimization of waterflooding problems[C].Intelligent 
Energy Conference and Exhibition. Amsterdam, The Netherlands, 2006

[3] ZHAO H., CHEN C. H. and DO S. et al. Maximizationof a dynamic quadratic interpolation model for production optimization[J]. SPE Journal, 2013, 18(6): 1012-1025.

[4] BROUWER D. R. Dynamic water flood optimization with smart wells using optimal control theory[D]. Delft, The Netherlands: Delft University of Technology, 2004.

[5] JANSEN J. D., DOUMA S. D. and BROUWER D. R. Closed-loop reservoir management[C]. SPE Reservoir Simulation Symposium, 119098. The Woodlands, Texas, USA, 2009.

[6] ZHANG Kai, LI Yang and YAO Jun. Theoretical research on production optimization of oil reservoirs[J]. Acta Petrolei Sinica, 2010, 31(1): 78-83(in Chinese).
[7] Kai ZHANG., Li-ming ZHANG., Jun YAO., Yu-xue CHEN., Ran-ran LU . Water flooding optimization with adjoint model under control constraints[J]. Journal of Hydrodynamics, Ser. B. 2014, 26(1): $75-85$

[8] Drosos Kourounis., Louis J. Durlofsky., Jan Dirk Jansen., Khalid Aziz. Adjoint formulation and constraint handling for gradient-based optimization of compositional reservoir flow $[\mathrm{J}]$. Computational Geosciences. 2014, 18(2): 117-137

[9] Eka Suwartadi., Stein Krogstad., Bjarne Foss. Nonlinear output constraints handling for production optimization of oil reservoirs[J]. Computational Geosciences. 2012, 16(2): 499-517 